\title{
The use of Doppler ultrasonography for pre- and post-surgery monitoring of abdominal aortic aneurysm. A case report
}

\author{
Monica Elia Georgescu1 ${ }^{\text {, Philippe Arbeille², Michaela Dobre }}{ }^{3}$, Victorita Stefanescu $^{3}$
}

${ }^{1}$ Centre Hospitalier Universitaire Trousseau, Tours, France, ${ }^{2}$ Department of Nuclear Medicine \& Ultrasounds, School of Medicine, University of Tours, ${ }^{3}$ Department of Morphological and Functional Sciences, Faculty of Medicine and Pharmacy, "Dunarea de Jos" University of Galati

\begin{abstract}
A 75 year old male patient was monitored for 3 years by Doppler Ultrasonography (US) for an abdominal aorta aneurysm (AAA). Because the aneurysm increased significantly, an aortic prosthesis was installed via an endovascular procedure. After one month of post-surgery monitoring, both Doppler US exam and contrast enhancement US (CEUS) suspected the presence of a leak at the level of the prosthesis. A new surgical procedure was scheduled and intraoperative arteriography confirmed an endoleak type II. Although not always able to specify the correct type of linkage, CEUS remains a reliable method for investigating the postoperative complications of AAA.
\end{abstract}

Keywords: abdominal aorta aneurysm, Doppler ultrasonography, prosthesis

\section{Introduction}

Each year, thousands of people die as a result of abdominal aorta aneurysm (AAA). Trends in male and female age-standardized AAA mortality vary from country to country. Literature data indicate a prevalence of AAA between $5-10 \%$ in men over 65 years [1]. The Romanian AAA mortality average is currently higher than that of most European country averages [2]. The most frequent cause is the decrease in wall elasticity due to a genetic defect [3], disturbance of the activity of the proteolytic enzymes [4], or development of atherosclerotic plaque [5]; less frequently, the origin might be related to an infectious or inflammatory process [6].

Given the very low risk of rupture when the aortic diameter is below $50 \mathrm{~mm}$ and the variable dynamic of the aneurysm growth, AAA patients require careful medical surveillance [7]. Doppler ultrasonography (US) is the exam of first choice in order to perform this surveillance, as it is a reliable and non-invasive technique for arterial

Received 05.06.2015 Accepted 25.07.2015

Med Ultrason

2016, Vol. 18, No 1, 127-130

Corresponding author: Michaela Dobre

35, Al. I. Cuza Street

800216, Galati, Romania

E-mail: mdobre@ugal.ro wall blood velocity quantification and is currently used for surgical decision indication. After surgery, Doppler US examination remains the easiest, most reproducible, and reliable technique for the prosthesis assessment and detection of a possible leak. In addition, contrast enhancement US (CEUS) indicates the exact location of leak, as an X-ray angiography, but without radiation [8] - but interpretation of the results must be done with caution and the decision to change the prosthesis should be taken only corroborating with angiography.

We present the case of a male patient with endovascular aneurysm/aortic repair (EVAR) which required an aorto-biiliac prosthesis, monitored pre- and post-surgery by Doppler US. In post intervention, CEUS suspected an anterograde injection of aneurysmal sac. The arteriography performed during surgery revealed the integrity of the prosthesis and aneurysmal sac re-injection through a low lumbar artery, avoiding a prosthesis change.

\section{Case report}

Between 2012 and 2014, a male patient aged 77 years old, was monitored for AAA using a Toshiba ISTYLE machine with an abdominal 2D probe of $3.5 \mathrm{MHz}$ (PVT $375 \mathrm{BT}$ ) and a 3D abdominal of 3.5 MHz (PVT375MV). The Doppler US exam, conducted every six months, showed the presence of an aneurysm of $20 \mathrm{~mm}$ diameter 


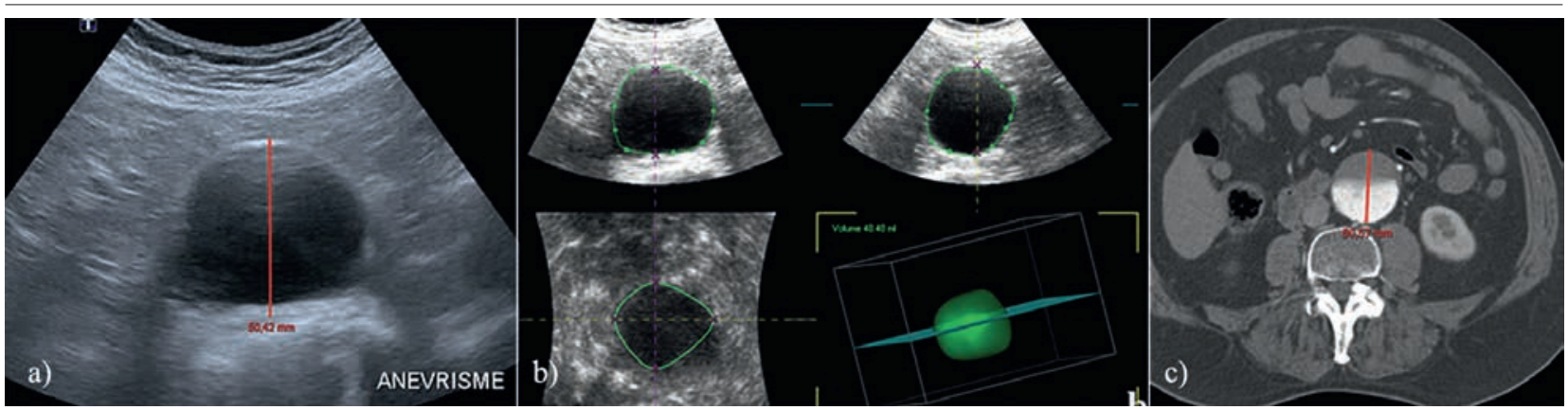

Fig 1. Imaging aspect of the aneurysm in evolution (2014): a) grey-scale US, transverse scan showing a maximum anteroposterior diameter of $50 \mathrm{~mm}$; b) 3D US indicated a volume of $48.48 \mathrm{~cm}^{3}$; c) computed tomography confirmed the increased diameter - $50.57 \mathrm{~mm}$.

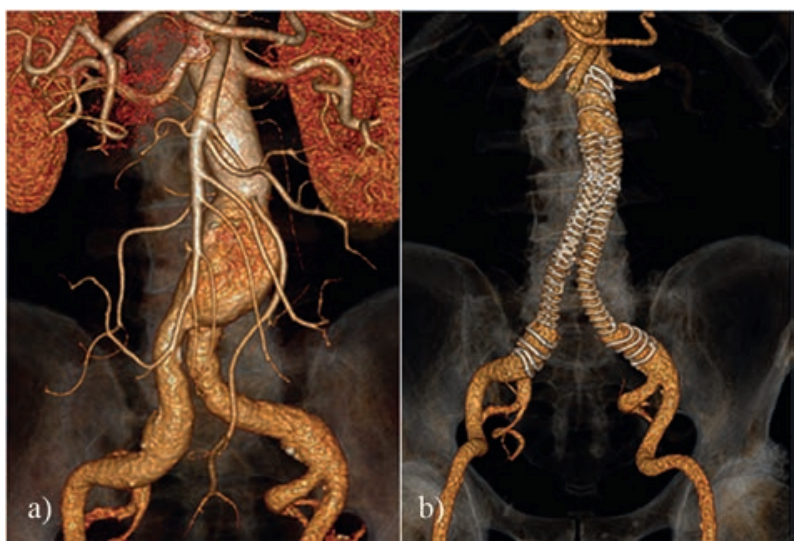

Fig 2. Computer tomography angiography aspects pre- and post-surgery: a) aneurysmal sac in the subrenal aorta; b) aortoiliac stent-graft (VASCUTEK endoprosthesis).

below the renal area, starting at the level of the inferior mesenteric: it increased from $36 \mathrm{~mm}$ to $50 \mathrm{~mm}$, requiring surgery (fig 1).

The angioscanner was performed before surgery in order to explore the entire thoracic and abdominal aorta searching for a possible associated thoracic aorta aneurysm and to check the size and locations of the AAA detected by Doppler US. The endovascular aneurysm/ aortic repair (EVAR) was performed at the end of 2014 at the University Hospital of Tours. A standard bi-iliac aortic endoprosthesis with sub-renal anchorage (VASCUTEK B30) was implanted without complications (fig 2). The prosthetic branches required two extensions, configured according to patient anatomical characteristics: $12 / 60+12-19 / 130$ on the left side (left iliac leg) and $12 / 80+12-21 / 130$ on the right side (right iliac leg).

The patient left hospital after a 3D ultrasound and conventional abdominal X-ray. A surveillance of the prosthesis by Doppler US was performed every month during three consecutive months, as well as by CT scan.

One month after surgery the Doppler US confirmed the existence of a leak (the aneurysmal sac raised from $49 \mathrm{~mm}$ after surgery to $70 \mathrm{~mm}$ at one month) as well as the presence of an arterial flux which entered the aneurysmal sac starting from a ramification of the endoprosthesis. The CEUS performed afterwards confirmed the leak (suggesting an endoleak type III, according to the White classification [9]), its location (left iliac leg of the prosthesis, anterior and median to the aneurysmal sac), and measured leak flow velocity at $66 \mathrm{~cm} / \mathrm{s}$ (fig 3). This type of endoleak is due to a defective graft fabrication or a poor sealing/junction between the modules of the prosthesis, which entails a subsequent complementary intervention (risk of rupture of aneurysmal sac) [10].

A new arteriography and implementation of an additional extension were proposed to the patient. Intraoperative arteriography evidenced a retrograde injection of aneurysmal sac through the left side of the prosthesis, at the junction with the left iliac extension (endoleak type II) (fig 4).

No intervention on the prosthesis was decided. Surveillance procedure was continued by echo-Doppler up to an eventual selective embolization - if necessary. Due to the accuracy of the CEUS examination in measuring the leak flow velocity, no new angioscan was requested along the surveillance, avoiding any additional exposure of the patient to radiation.

\section{Discussions}

The endovascular treatment of AAA is increasingly preferred both by medical specialists and patients because of the distinct advantages (shorter operative time and hospitalization, no general anaesthesia, decreased trauma and bleeding), despite the potential risks of incomplete AAA sealing, aneurysm increasing, or graft thrombosis [10-12]. Leakage risk requires a careful follow-up after EVAR. According to French hospital protocol, surveillance is performed at least once a month during the first three months after intervention. 


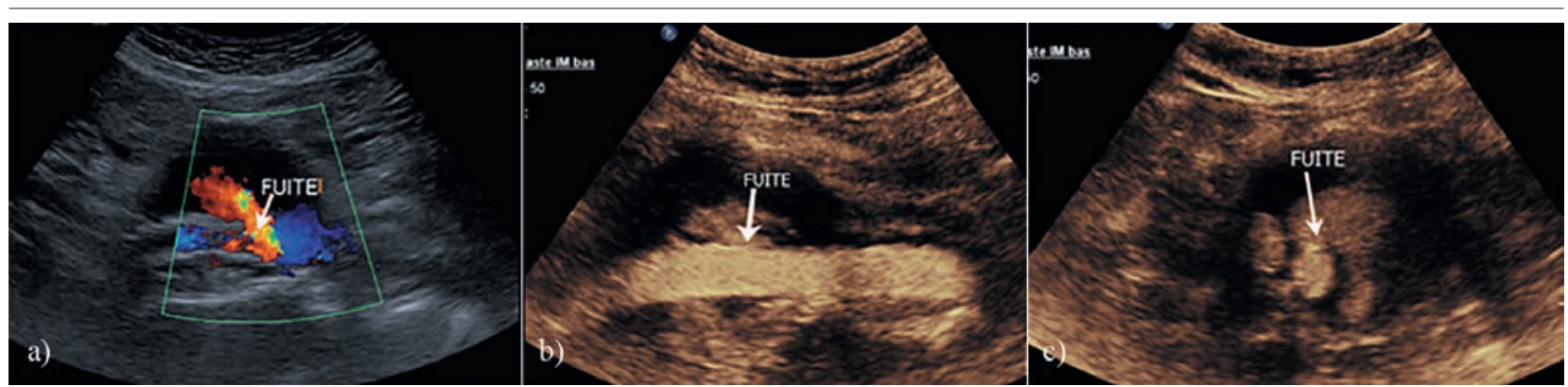

Fig 3. Doppler and CEUS of the endoleak: a) abnormal flux in the aneurism sac (arrow); b) longitudinal section on the prosthesis and the aneurysmal sac, viewing the opacification of the prosthesis as a result of endoleak - hyperechogenicity results of blood presence on the outside of prosthesis (arrow); c) transverse scan at aortic bifurcation prosthesis highlighting a leakage from its left extension, with hyperechoic area on aneurysm sac (arrow).

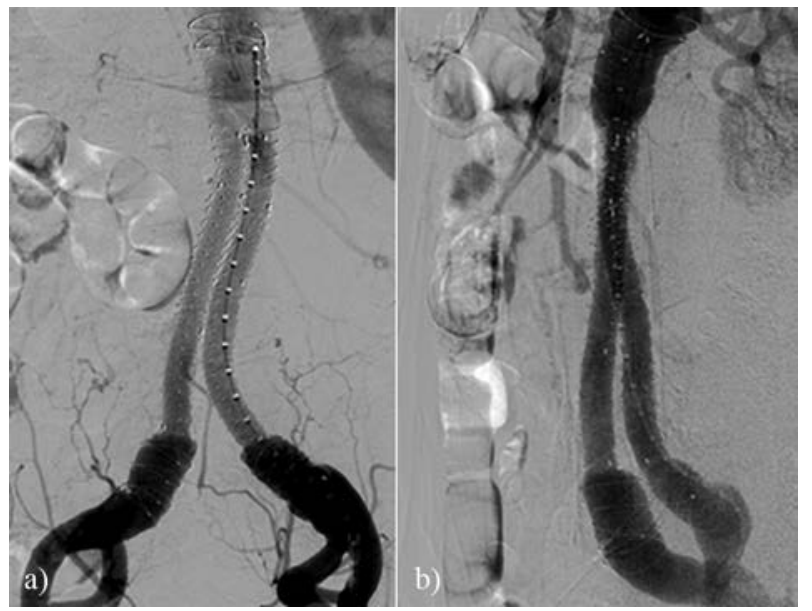

Fig 4. Intraoperative arteriography: a) front view of the prosthesis. The integrity of the prosthesis removes the suspicion on type I or III leakage; b) lateral view. The retrograde injection of the aneurysmal sac via lumbar artery indicates type II endoleak.

Our case reveals both the contribution and the difficulties in determining the cause of leakage in EVAR Doppler US monitoring. The type III leakage, as initially suspected, depends on a defect of the prosthesis material. Such a kind of leakage has become very rare considering the producers' concern in product improvement [13-18]. An endoleak type II is the result of the injection of aneurysmal sac through a lumbar or mesenteric artery - which often does not require reintervention because it spontaneously resolved in time [19]. In our case, because the injection of the aneurismal sac was produced by a low lumbar artery, starting right at the left iliac bifurcation of the endoprosthesis, the leakage was visualised by CEUS as anterograde, starting at the left graft extension, therefore supposing an endoleak type III. The arteriography, allowing front and profile views of the prosthesis, revealed its integrity and confirmed the endoleak type II. Thereafter, CEUS assume the role of monitoring, by pe- riodically measuring the dimensions of the aneurysmal sac, which correlate the persistence of leakage.

In the present case, the Doppler US and CEUS surveillance post-EVAR were sufficient to diagnose the presence of a leak at the level of the prostheses and to quantify the arterial flow inside the leak, as affirmed in similar recent literature reports [20-23]. Informations about the haemodynamic of the leak are of particular interest as a high flow leak requires immediate treatment.

Actually standard echography is considered as insufficient for direct use but CEUS has reliability and specificity to be a correct alternative to CT angiography [21,22]. Only if we consider that CEUS avoids potential induced nephropathies caused by repeated ionizations, the alternative is suitable. In our case the use of CEUS was reliable and specific for the aneurysmal sac for endoleak identification. The type of endoleak is the most important criteria to define if the monitoring is continuing (type II) or if a new intervention is compulsory (type III). In the last ten years the performance of CEUS is generally accepted as an optimal alternative to other invasive methods [23].

Our practical conclusions are in line with international opinion on the subject [11]: the performance could be obtained only with top quality technical conditions associated with high costs; the high level instruments have to be manipulated by highly trained and experienced experts collaborating with all teams involved in AAA treatment; the senior experts have to be involved in patient selection; in some cases poor echogenicity could lead to the elimination of US methods or necessity of a permanent check of image quality confirmed by CT.

As a final conclusion, CEUS is a reliable method for the investigation of postoperative complications of AAA even it is associated with higher costs related to material and expertise. In time it could be necessary to establish some common protocols at an international level in order to cover all qualitative aspects of investigations. 


\section{References}

1. Crişan S, Militaru V, Crişan IM. Abdominal aortic aneurysm - online methods of patient education. Med Ultrason 2010; 12: 22-25.

2. Sidloff D, Stather P, Dattani N, et al. Aneurysm global epidemiology study: public health measures can further reduce abdominal aortic aneurysm mortality. Circulation 2014; 129: 747-753.

3. Golledge J, Kuivaniemi H. Genetics of abdominal aortic aneurysm. Curr Opin Cardiol 2013; 28: 290-296.

4. Sorimachi H, Ono Y. Regulation and physiological roles of the calpain system in muscular disorders. Cardiovasc Res 2012; 96: 11-22.

5. van den Borne P, van der Laan SW, Bovens SM, et al. Leukotriene B4 levels in human atherosclerotic plaques and abdominal aortic aneurysms. PLoS One 2014; 9: e86522.

6. Ishizaka N, Sohmiya K, Miyamura M, et al. Infected aortic aneurysm and inflammatory aortic aneurysm - In search of an optimal differential diagnosis. J Cardiol 2012; 59: 123131.

7. Zhao S, Li W, Gu L. Biomechanical prediction of abdominal aortic aneurysm rupture risk: Sensitivity analysis. JBiSE 2012; 5: 664-671.

8. San Norberto EM, Taylor J, Vaquero C. Color-Doppler U1trasonography in the Monitoring of Endovascular Abdominal Aortic Aneurysm Repair. In: Grundmann R. (ed.). Diagnosis and Treatment of Abdominal and Thoracic Aortic Aneurysms Including the Ascending Aorta and the Aortic Arch. Rijeka, InTech 2011: 37-56.

9. White GH, Yu W, May J, Chaufour X, Stephen MS. Endoleak as a complication of endoluminal grafting of abdominal aortic aneurysms: classification, incidence, diagnosis and management. J Endovasc Surg 1997; 4: 152-168.

10. Moll FL, Powell JT, Fraedrich G, et al. Management of abdominal aortic aneurysms clinical practice guidelines of the European society for vascular surgery. Eur J Vasc Surg 2011; 41(Suppl 1): S1-S58.

11. Long A, Louail B, Turmel-Rodrigues L, Julia P, Sapoval M. EVAR surveillance: is ultrasound imaging alone reliable in routine practice? In: Becquemin JP, Alimi YS, Wateletbe J. (eds). Controversies and updates in vascular surgery. Turin, Edizioni Minerva Medica 2005: 8-16.

12. Deklunder G, Sediri I, Donati T, Boivin V. Gautier C, Haulon S. Intérêt de l'échographie de contraste dans la surveil- lance des endoprothèses aortiques. J Radiol 2009; 90: 141147.

13. Kwolek C, Moore R. One year Results of the Vascutek Anaconda $^{\text {TM }}$ One-Lok EVAR trial. Presented at: Vascular Annual Meeting, 2014 June 5-7, Boston, MA, USA. Accessed May 2015.

14. Pratesi G. Preliminary 3-Year European results with a lower profile device for EVAR: the INNOVATION Study of the INCRAFT AAA stend-graft. Presented in: VEITHsymposium, 2014 Nov. 18-22, New York, USA. [online]. Accessed May 2015. http://www.veithsymposium.org/abstracts/2014/vei/609.pdf

15. Verma H, Meda N, Tripathi RK. Management of Complex Type IIIa Endoleak by Brachio-femoral Realignment and Interposition Stent Graft and Review of the Literature. Indian J Vasc Endovasc Surg 2014; 1: 29-32.

16. Rascanu C, Jansen B, Giannakidis A, Bitlisli B, Klenk E. Considerații privind tratamentului endovascular al anevrismelor aortice infrarenale. Chirurgia 2011; 106: 445-450.

17. Debing E, Aerden D, Gallala S, Vandenbroucke F, Van den Brande P. Stenting complex aorta aneurysms with the Cardiatis multilayer flow modulator: first impressions. Eur J Vasc Endovasc Surg 2014; 47: 604-608.

18. ***Premiera mondiala la Sibiu: operatie revolutionara pentru anevrism aortic. Stiri de Sibiu 14.04.2014. Accessed May 2015. http://www.stiridesibiu.ro/?p=4095

19. Tolia AJ, Landis R, Lamparello P, Rosen R, Macari M. Type II endoleaks after endovascular repair of abdominal aortic aneurysms: natural history. Radiology 2005; 235: 683-686.

20. Franca GJ, Baroncini LAV, Oliveira Ad, et al. Evaluation with Doppler vascular ultrasound in postoperative endovascular treatment of abdominal aortic aneurysm: a prospective comparative study with angiotomography. J Vasc Bras 2013; 12: 102-109.

21. Abbas A, Hansrani V, Sedgwick N, Ghosh J, McCollum CN. 3D contrast enhanced ultrasound for detecting endoleak following endovascular aneurysm repair (EVAR). Eur J Vasc Endovasc Surg 2014; 47: 487-492.

22. Sarlon G, Lapierre F, Sarlon E, Bartoli MA, Magnan PE, Branchereau A. Surveillance des endoprothèses aortiques abdominales: intérêt de l'échographie-doppler standard et avec contraste. J Mal Vascul 2009; 34: 34-43.

23. Sun Z. Diagnostic value of color duplex ultrasonography in the follow-up of endovascular repair of abdominal aortic aneurysm. J Vasc Interv Radiol 2006; 17: 759-764. 